\title{
Cécile: \\ Fürstenmätresse in bürgerlicher Zeit
}

Viele Romane Theodor Fontanes haben als Keim ein tatsächliches Ereignis, von dem der Autor aus Zeitung, Brief oder Gespräch zufällig erfuhr und das ihn in seiner Einbildungskraft dann lange beschäftigte, bis es sich mit anderen Handlungs- und Bedeutungselementen verband und $\mathrm{zu}$ einem regelrechten Romanvorwurf auswuchs.

Auch Cécile verdankt seinen Ursprung wohl einer solchen Mitteilung. Anfang 1882 notiert sich Fontane nach einer Unterhaltung mit dem Grafen Philipp von Eulenburg »die Geschichte mit seinem zweiten Sohn, die [...] nahe daran war, zu einem Duell zwischen dem Obersten Graf Alten und dem Lieutenant Graf Eulenburg zu führen. Dieser wollte heiraten und berichtete davon dienstgemäß seinem Vorgesetzten, der zu seiner Überraschung erwiderte: "Lieber Eulenburg, solche Damen liebt man, aber heiratet man nicht. « Das Duell, zu dem der Leutnant den Obersten herausforderte, wurde nur dadurch vermieden, daß Graf Alten die Angelegenheit dienstlich behandelte. Anders als Fontanes Cécile von St. Arnaud aber wurde die >Dame< schließlich mit Graf Eulenburg glücklich. Das Paar hatte auch mehrere Kinder.

Es dauert etwa 4 Jahre, bis sich aus diesem Keim der fertige Roman entwickelt, in dem dieses Ereignis nur noch als Episode aus der Vergangenheit der Heldin in das Geschehen hineinragt und es überschattet. Den 1. Entwurf des Werks schreibt Fontane im Juli 1884 in Thale im Harz nieder, wo die ersten 16 Kapitel der Handlung spielen, und damit ist ein zweites für Fontanes Arbeitsweise typisches Element genannt: Der Autor erforscht den realen Handlungsrahmen seiner Bücher sorgfältig und lernt ihn gerne selber kennen, wobei er sich auch vor eigens dazu unternommenen Reisen nicht scheut. »Ich $m u ß$ die letzten drei Juni-Wochen in Thale zubringen, weil ich dort - im ersten Entwurf - eine Novelle niederschreiben will, deren erste Hälfte in Thale im Hotel Zehnpfund spielt. Ich muß das Lokal vor Augen [...] haben ${ }^{2}{ }^{2}$ schreibt er im Bezug auf Cécile am 5. 6. 1884 an Emilie Zöllner, und noch genereller heißt es am 3. 6. 1885 an seine Frau: "Ich habe nicht die Frechheit drauf los zu schreiben, ohne Sorge darum, ob es stimmt oder nicht. ${ }^{3}$ Zwar wohnt Fontane bei seinem Besuch im Sommer 1884 nicht im Hotel Zehnpfund, das er aber von früheren Aufenthalten her kennt, doch die Briefe an seine Frau geben Auskunft darüber, wie er Bekannte, Situationen, Eindrücke und Schauplätze für den entstehenden Roman verwendet. Menschen, mit denen er seine Tage verbringt, geben Züge zu einzelnen Figuren her, und das Äußerliche des alten Präzeptors von Altenbrak und seiner Tochter, der Förstersfrau, geht fast 
unverändert in den literarischen Text ein. Fontane besucht $u$. a. auch Quedlinburg und schreibt am 22. 6. 1884 an seine Frau: »Heut abend werde ich Schmerlen essen, meiner neuen Novelle zuliebe $[\ldots] . \ll^{4}$

Den Spätsommer desselben Jahres verbringt Fontane im Riesengebirge, wo dann später zum Teil sein Roman Quitt spielen wird, und bei diesem Urlaub wird Cécile im wesentlichen ausgearbeitet. Der Harzer Entwurf von 14 Kapiteln wächst nun wohl zu den 22 Kapiteln an, die er nach Fontanes eigener Auskunft in einem Brief an Adolf Glaser vom 22. 4. 1885 damals hat. Das "Aufputzen und Striegeln ${ }^{5}$ des Textes, von dem im selben Brief die Rede ist und das vor allem im Herbst dieses Jahres stattfindet, muß also noch erhebliche Veränderungen mit sich gebracht haben, damit das Manuskript den endgültigen Umfang von 29 Kapiteln erreicht.

Im folgenden Jahr 1886 erscheint Cécile als Vorabdruck in der Zeitschrift Das Universum, mit der Fontane vorher noch keinen Kontakt gehabt hat. Der Redakteur hat ihn um einen Roman gebeten und zahlt zur Freude des Autors prompt. Die Buchausgabe bereitet größere Schwierigkeiten. Erst der 3. Verleger, dem Fontane Cécile anbietet, bringt das Buch heraus, dessen Erscheinen sich dadurch bis April 1887 hinzieht.

Cécile steht damit zeitlich und numerisch etwa in der Mitte von Fontanes Romanwerk: 7 Romane erscheinen davor, 8 folgen; der erste, Vor dem Sturm, erscheint 1878, der letzte, Der Stechlin, als Buch erst kurz nach Fontanes Tod 1898. Aber man darf natürlich nicht außer acht lassen, daß die Romanproduktion in Fontanes Gesamtschaffen nur die letzten beiden Jahrzehnte ausfüllt. Als Cécile vorliegt, geht der Verfasser auf die $70 \mathrm{zu}$, und doch findet er unter Krisen, die durch die Vorstellung vom sheiteren< Fontane zu sehr aus dem öffentlichen Bewußtsein verdrängt worden sind, erst jetzt das literarische Genre, für das sein Name in der deutschen Literatur des 19. Jahrhunderts steht: den Zeitroman als »Vielheitsroman«, der die Zeitanalyse im kunstvoll komponierten Personenensemble und in der Vielfalt sich spiegelnder und wechselseitig deutender, vielfach in der Großstadt Berlin handelnder Situationen erstrebt. Damit entfernt sich der Autor von der spezifisch deutschen Tradition des Bildungsromans, der die innere Reifung eines Menschen an meist peripheren Schauplätzen darstellt - die Großstadt kommt schon im ersten groBen Bildungsroman, in Goethes Wilhelm Meister, nicht vor. Fontane erobert sich mit diesem in Deutschland auf dieser literarischen Höhe konkurrenzlosen Romantyp spät im Leben als einziger deutscher Romancier des 19. Jahrhunderts seinen Platz in der Reihe der großen europäischen Romanschriftsteller dieser Zeit. 
Zum Verständnis Céciles hat Fontane selbst in 2 Briefen Hinweise gegeben, die aber mehr Fragen aufwerfen als beantworten. Adolf Glaser, einem Redakteur von Westermanns Monatsheften, gegenüber charakterisiert er den Roman folgendermaßen: »Stoff: Ein forscher Kerl, $35,{ }^{6}$ Mann von Welt, liebt und verehrt - nein, verehrt ist zuviel - liebt und umkurt eine schöne junge Frau, kränklich, pikant. Eines schönen Tages entpuppt sie sich als reponirte Fürstengeliebte. Sofort veränderter Ton, Zudringlichkeit mit den Allüren des guten Rechts. Conflikte; tragischer Ausgang. $\aleph^{7}$ Dem Kritiker Paul Schlenther schreibt Fontane nach dem Erscheinen der Buchausgabe und der ersten Rezensionen: "Cécile ist doch mehr als eine Alltagsgeschichte, die liebevoll und mit einem gewissen Aufwande von Kunst erzählt ist. Wenigstens will die Geschichte noch etwas mehr sein. Sie setzt sich erstens vor, einen Charakter zu zeichnen, der, soweit meine Novellenkenntnis reicht (freilich nicht sehr weit), noch nicht gezeichnet ist, und will zweitens den Satz illustrieren: > wer mal drin sitzt, gleichviel mit oder ohne Schuld, kommt nicht wieder heraus.< Also etwas wie Tendenz. $\ll^{8}$

In beiden Fällen beziehen sich die Bemerkungen auf Handlung, Hauptpersonen und Moral oder, wie Fontane sagt, "Tendenz « der Geschichte und betonen damit Aspekte, die aufschlußreich sind für das literarische Selbstverständnis der 2. Hälfte des 19. Jahrhunderts. Es ist eine Zeit, in der vor allem die weibliche Leserschaft Belletristik konsumiert und Romanheldinnen schätzt, bei denen man mitleiden kann. Fontane findet demgemäß, daß der Wert seiner Erzählung dadurch steigt, daß der Typ der Titelheldin den Reiz der Neuheit hat. Aber zweifellos verharmlost der Autor selbst damit die Substanz seines Werkes, und der "gewisse Aufwand von Kunst« wäre unter dieser Voraussetzung verschwendet. Nichts an Fontanes eigener Charakteristik des Werkes ist spezifisch >Fontanisch<, und weist auf die Bedeutung hin, die der Romancier heute als Künstler und Zeitanalytiker genießt. Wie kommt das?

Man muß sich die literatursoziologische Situation der Zeit vergegenwärtigen, um Fontanes >understatement ‘, das über den künstlerischen und geistigen Rang seines Werkes hinwegtäuscht, zu verstehen. Fontane ist bis zu seinem Tode im Alter von fast 80 Jahren auf die finanziellen Erträge aus seinen literarischen Arbeiten angewiesen. Er hat 1876, als er den Posten des Sekretärs der Akademie der Künste in Berlin aufgibt, bewußt und in nervenbelastenden Auseinandersetzungen mit seiner Frau die Entscheidung gefällt, schriftstellerische Freiheit und Unabhängigkeit mit finanzieller Unsicherheit und Beschränkụng zu erkaufen. Aber während er so über seine Zeit verfügen kann und sich dienstlicher Pflicht und beruflicher Unterordnung entzieht, begibt er sich in die Abhängigkeit des literarischen Marktes und muß sich - je- 
denfalls äußerlich - anpassen. Entsprechend muß er vom eigentlichen Gehalt seiner Werke und von seiner subtilen schriftstellerischen Technik - beides erschließt sich zum großen Teil erst im 20. Jahrhundert der Leserschaft und der Forschung - eher ablenken, wenn er auch ohne >Bestseller «, der ihm vor Effi Briest (1894) versagt ist, im Geschäft bleiben will. Seine Äußerungen über seine Bücher sind häufig von dem Wunsch bestimmt, einen Verleger zu finden und ihm das neue Werk als möglichen Zeitschriften- oder Bucherfolg schmackhaft zu machen oder den Roman für einen Kritiker in den zeitgenössischen literarischen Kontext einzuordnen.

Dieser Markt aber wird beherrscht von den Familienzeitschriften, deren große Zeit diese 2. Hälfte des 19. Jahrhunderts ist und deren spießbürgerliche Gemütlichkeit wir heute belächeln: Die Gartenlaube, Daheim, Nord und Süd, Vom Fels zum Meer usw. Obwohl manche von ihnen durchaus bürgerlich-liberal und daher im Zeitkontext eher progressiv sind, müssen sich die darin in Fortsetzungen erscheinenden belletristischen Werke engen bis prüden Moralvorstellungen unterwerfen, soziale Schattenseiten der Gesellschaft und politische Kritik meiden und Romangestalten anbieten, die starke Gefühlsreaktionen beim Publikum auslösen. Daß Fontane, dessen sexuelle Diskretion und sozialkritische Indirektheit zu den auffallenden Merkmalen seiner Kunst gehören, trotzdem immer wieder Schwierigkeiten hat, eine Zeitschrift zu finden, die sein jeweils neues Werk abdrucken will, oder daß der Vorabdruck auf heftige Ablehnung bei einem Teil des Publikums oder beim Verleger selbst stößt, zeigt, wie eng die Grenzen für den Roman der Zeit sind, wenn der Autor unter Publikationszwang steht. Wie Fontane diese Beschränkungen unterwandert hat, ist Teil seiner Größe; wie er es im Fall Cécile getan hat, wird gleich erkennbar werden. Da sich durch den Vorabdruck seiner Romane und die dann folgende Buchausgabe die Einnahme verdoppeln läßt, kann Fontane es sich nicht leisten, den Zeitschriftengeschmack außer acht zu lassen. Tatsächlich werden seine $16 \mathrm{zu}$ Lebzeiten erschienenen Romane - der 17., Mathilde Möhring, kommt erst aus dem Nachlaß ans Licht - allesamt in Zeitschriften vorabgedruckt, und zwar insgesamt in 10 verschiedenen - eine Zahl, die allein schon ein Schlaglicht auf seine Probleme wirft.

Die Kehrseite dieser erzwungenen Anpassung bildet denn auch Fontanes immer wieder ausbrechende Verachtung für die Seichtigkeit des Geschmacks und die Oberflächlichkeit des lesenden Publikums, seine Verzweiflung über das Versagen der Kritik (»Nichts liegt hier so danieder, wie die Kritik «) und die künstlerische Blindheit der Zeitgenossen, sein Ärger über die Prätentionen weniger begabter, aber besser verdienender Schriftstellerkollegen. Immer wieder möchte er am liebsten alles hinwerfen und beklagt sich über das »literarische Sechsdreiertum «. ${ }^{10}$ Fontane war sich seines künstlẹischen Ranges durchaus bewußt. Erst wenn man also durch die von ihm selbst eher verschleiernd beschriebene Oberfläche seiner Romane dringt, enthüllt sich deren 
künstlerische Gestalt und deren leidenschaftlicher Zeitkommentar. Entsprechend seinem Motto $"$ Der Zauber steckt immer im Detail ${ }^{11}$ sind diese Dimensionen seiner Werke im Text verborgen und müssen durch sorgfältige Analyse erschlossen werden. Mit Recht hat Fontane einmal »auf die hundert und, ich kann dreist sagen, auf die tausend Finessen $\aleph^{12}$ hingewiesen, die in seinen Romanen stecken.

Das Charakteristische an Fontanes Romanen ist ihre Doppelbödigkeit. Was wie schlichte Wiedergabe der Wirklichkeit erscheint, ist ein dichtes Gewebe von indirekten Kommentaren zum Geschehen, die dessen eigentliche Bedeutung enthalten. Die sinnenhaft erfahrbare und detailliert beschriebene Realität wird zum Medium von Bedeutung. So entsteht eine Struktur von 2 Ebenen, für die jüngst Peter-Klaus Schuster einen Terminus aus der bildenden Kunst vorgeschlagen hat: »disguised symbolism ${ }^{13}{ }^{13}$ versteckte Symbolik. In Gestalten, Handlungsorten und -elementen, in Gegenständen und erwähnten Kunstwerken oder historischen Figuren, in Zitaten und Namen sind Sinn und Botschaft der Bücher, oft, auf subtilste Weise maskiert, eingelagert. Es ist dabei durchaus möglich, Fontanes Romane mit Vergnügen zu lesen, ohne irgend etwas von dieser Doppelbödigkeit zu merken.

Zeitkommentar - um an einer bloßen Kleinigkeit aus Cécile zu erläutern, was hier gemeint ist - ist daher in Fontanes Werken auch das harmloseste und unscheinbarste Detail und es ist nicht übertrieben zu behaupten, daß die Durchdringung der Fontaneschen Texte noch nicht weit fortgeschritten ist. Daß etwa in Thale, wohin Cécile wegen der guten Luft geht (»Thale, klimatischer Kurort«), nun eine Fabrik die Luft verpestet, zeigt, wie wenig Aussicht zur Heilung der kranken Frau besteht und wie sehr ihre Krankheit selbst mit dem Wandel der Zeit zu tun hat; daß aber diese Fabrik an die »Kuhnheimsche Fabrik in Berlin erinnert und ausgerechnet »Blech mit Emaille« (17) produziert, also wertloses Metall, schön und glatt überzogen, ist ein Kommentar auf die moderne Zeit überhaupt und bildet ein Detail in einem Motiv des Buches: der frischen Luft. Gordons Persönlichkeit etwa enthüllt sich dem Leser schon darin, daß er zuviel Luftzug haßt und sich damit indirekt als Feigling beschreibt.

Betrachtet man Cécile unter solcher Perspektive, sucht man durch die Oberfläche zu dringen, dann erschließt sich das Buch als Zeitanalyse und -kritik, dann erweist es sich wie fast alle Romane Fontanes als kritischer Kommentar auf das Preußentum seiner Zeit, dann geht es weit über die Darstellung des tragischen Schicksals einer verwöhnten, gedemütigten und nervlich kranken Frau hinaus, die von ihrer Vergangenheit verfolgt wird. In Wirk- 
lichkeit nämlich stoßen in Cécile von St. Arnaud und den beiden Männern, die sie umgeben, St. Arnaud und Gordon, 2 Welten aufeinander, die unvereinbar sind und um deren Charakterisierung es Fontane eigentlich geht. Wie ihre bekanntere Leidensgenossin Effi Briest - auch sie muß sexuelle Verstöße gegen die Konventionen der Zeit büßen - ist auch Cécile ein Opfer der Gesellschaft, ein Opfer Preußens. In Effi Briest ist diese Botschaft an den Leser unter anderem darin enthalten, daß die Titelheldin nach ihrer Scheidung in der Königgrätzer Straße wohnt, benannt nach dem Ort, wo Österreich 1866 vernichtend von Preußen geschlagen wurde. Daß Effi jetzt nicht mehr zu den Preußen, sondern zu den geschlagenen Österreichern gehört, wird noch deutlicher, als sie sich das Essen aus dem »Habsburger Hof« kommen läßt, während sie zur Zeit ihrer Ehe in Kessin in einem Gasthof mit dem Namen »Zum Fürsten Bismarck « gefrühstückt hat. Ohnehin ist auf versteckte Weise Bismarck die zerstörerische Kraft hinter ihrer Ehe, ein Aspekt, der auch in Cécile nicht übersehen werden darf. Es ist gewiß kein Zufall, daß Gordon durch die Königgrätzer Straße und über den Potsdamer Platz zur Wohnung der St. Arnauds am Hafenplatz geht, wie Cécile Gordons romantisch-exotischen Erwartungen schon vom Haus her nicht ganz entspricht, denn sie wohnt nicht in dem »Haus mit der Alhambrakuppel, sondern [in] ein[em] benachbarte[n] von kaum minderer Eleganz« (118). Auch alle diese Elemente sind wieder Beispiele für Fontanes versteckte Symbolik. Wie Effi kann auch Cécile - deren Vornamen dieselben, wie Fontane empfand, vornehmen $>i<-$ und $>$ e<-Laute enthält - in der Welt, in der sie zu leben gezwungen ist, nur zugrunde gehen, und schon ihr ständiges Kranksein ist Symptom dafür: Sie kränkelt an der Zeit selbst. Wie Effi von den zu vielen Siegen Preußens redet, so macht schon Céciles erste, außerordentlich sprechende Geste in dem Roman deutlich, wie sie zur Macht und zu den Siegen Preußens steht. Als der Zug nach dem Harz aus Berlin abfährt, ragt in der Ferne »halb gespenstisch « die Siegessäule auf: „Die Dame wies kopfschüttelnd mit der Schirmspitze darauf hin [...].« (8) $\mathrm{Daß}$ die erste Station dieser Reise das Zentrum des Preußentums, Potsdam, ist, wo die St. Arnauds geschnitten werden, verstärkt den Eindruck, daß Cécile und das Preußentum Gegner sind. Die Reise in den Harz ist eine Flucht vor Preußen, die Züge zurück nach Berlin scheinen vom Hexentanzplatz zu kommen und jagen ihr Unbehagen ein, aber gerade in der Entfernung von Berlin stellt sich heraus, daß Preußen und die bürgerliche, wenn nicht bourgeoise Welt, die es vertritt, überall ist. Gerade die Beschwörung der alten Kaiserpracht im Harz (»Hier [...] predigt alles Kaisertum und Kaiserherrlichkeit«, 69) macht um so auffälliger, wie sich die Zeit gewandelt hat; gerade Eginhards Askanierkult macht den Sieg der Hohenzollern um so deutlicher. Es ist wiederum eine Geste voller Bedeutung, daß die beiden Berliner Touristen, die das Preußisch-Berlinische so eindringlich gegenwärtig halten und sich dementsprechend in dem Buch gleich mit militärischem Jargon einführen 
(»Wo kampieren wir? [...] Aber erst hier das alte Schlachtfeld abräumen«, 16), sich mit dem Fernrohr die Türme von Halberstadt und Quedlinburg näher heranholen: Halberstadt, alte Hauptstadt eines selbständigen Fürstentums, und Quedlinburg, reichsunmittelbare Residenz der Fürstäbtissin, gehören schon lange zu Preußen; auch dort kann Cécile der neuen Zeit nicht entgehen. Sie aber macht einen Ausflug dorthin, während die Berliner als moderne Touristen sich auf den indirekten Kontakt mit dem Ferienziel durch das Fernrohr beschränken. Preußen ist auch der Bezugspunkt so vieler Gestalten des $\mathrm{Bu}$ ches. Was nämlich die meisten von ihnen gemeinsam haben, ist zweierlei: Erstens haben sie sich nahezu alle aus ihrer öffentlichen Tätigkeit zurückgezogen oder sind entlassen worden: Gordon und St. Arnaud haben den militärischen Dienst quittiert, und beide haben dabei finanzielles Mißgeschick. Gordon hat seine militärische Karriere wegen Schulden aufgegeben, und St. Arnaud wird aus guten Gründen der »Jeu-Oberst« (130) genannt. Beide lassen sich also mit der neuen Zeit ein, deren charakteristischstes Element das Geld ist, aber beide beherrschen dabei offenbar das Metier nicht richtig. Genau dieses Verraten alter Standesbegriffe und gleichzeitige Schlechtangepaßtsein an das Moderne kennzeichnet auch ihren Umgang mit Cécile und führt zur Katastrophe. Der Emeritus ist von seiner Gemeinde »um Mümmelns wegen« (28) entlassen worden; der Privatgelehrte ist eben Privatgelehrter; Geheimrat Hedemeyer ist »schon unter Mühler >kaltgestellt« (134) worden, und der alte Rodensteiner ist aus Stolz in den Ruhestand getreten. Während sich aber darin ihre Opposition oder mindestens ihr Abstand zu Preußen ausdrückt, sind sie doch zweitens gerade mit Preußen in starkem Maße beschäftigt und bestätigen dadurch den Einfluß und die Allgegenwärtigkeit des Hohenzollernreiches und des preußischen Geistes. Die Gesellschaft beim Diner der St. Arnauds besteht nur aus Preußen-Frondeurs, aber das Gespräch während des ganzen Abends dreht sich kaum um etwas anderes als gerade Preußen. Sie sind alle Preußen wider Willen, und diese scheinbare Distanz deuten schon die Namen an, die das Preußisch-Deutsche absichtlich meiden: St. Arnaud ist französisch, und da in Céciles Mädchennamen Woronesch von Zacha »eine ganze slawische Welt harmonisch zusammenklingt« 145), scheint sie westlich und östlich von Deutschland zu Hause zu sein, nicht dort selbst. Das Slawische deutet bei Fontane häufig auf Vitalität, Sexualität oder gar Chaotisches hin, und es stimmt durchaus mit diesem Eindruck überein, daß Eginhard Aus dem Grunde, der eigentlich Genserowsky heißt und aus Polen stammt (77), »etwas von einem Faun hatte (97); Gordon stammt aus Schottland, hat aber »den lebhaften Wunsch, in preußischen Dienst zurückzutreten « (58). Der Rodensteiner wollte einen prestigereicheren Orden, wollte also mehr von Preußen anerkannt werden, nicht weniger. Hedemeyer hat "seine Wiederanstellung [...] trotz andauernder Falk-Umschmeichlung nicht durchgesetzt « (134), und der Privatgelehrte spricht im Zusammenhang mit den Askaniern ständig von den 
Hohenzollern, zu denen sich der Emeritus offen bekennt, der treffenderweise die Gegenwart als »Zeitalter Otto von Bismarcks « (80) bezeichnet. Wenn sich in diesem Zusammenhang die Diskussion der beiden letzten um die Treue gegen das Alte und die Treue gegen das Neue dreht, wird in diesem Begriff die Beziehung zwischen der Zeit und dem privaten Schicksal Céciles deutlich, denn Gordon wird Cécile untreu und bekennt: »Wir bleiben unsrer Natur getreu, das ist unsre einzige Treue« (173), während Cécile ihre alte Treue gegenüber Menschlichkeit und Liebe zuletzt noch dadurch bestätigt, daß sie in Cyrillenort beigesetzt werden möchte. Wie nahe geht ihr die Hundetreue, über die man sich bei der Quedlinburger Schloßbesichtigung unterhält.

Mit diesem Gedanken ist ein weiteres Motiv des Romans verbunden, das Gordons Schwanken zwischen der alten und der neuen Haltung, zwischen dem ritterlichen Dienst an der Frau, bei dem die Liebe über die bürgerliche Moral siegt, und dem rigorosen Gut und Böse der bürgerlichen Konventionen, das den Menschen opfert um des Prinzips willen, charakterisiert: Wiederholt wird die mittelalterliche Welt metaphorisch beschworen. Gordon etwa wird von St. Arnaud ein »Chevalier errant« (58) genannt, ein irrender Ritter, der zuletzt »unritterlich und ehrlos« (177) gehandelt habe. Daß St. Arnaud den aristokratischen Ehrbegriff selbst pervertiert, beweist, daß auch er vom Dienst an der Frau nichts mehr versteht. Grausam sind sie beide und gehen im wahrsten Sinn des Wortes über Céciles Leiche, weil sie sich zuletzt in einem Kampfritual gegenüberstehen, bei dem die Frau keine Rolle mehr spielt. Diese menschenverachtende Grausamkeit, die unter der scheinbar so moralischen und zivilisierten Oberfläche der Gesellschaft herrscht, zieht selbst in eindringlichen Bildern durch das Buch. Hierher etwa gehören Wereschtschagins Bilder, auf deren einem die Tempelwächter sich lausen; hierher gehört die Erzählung der Baronin Snatterlöw, daß »persische Minister hier im Königlichen Schlosse [...] eine ganze Reihe von Hämmeln eigenhändig geschlachtet und die Schlachtmesser an den Gardinen abgewischt haben« (133). Wohl sind es Leute aus Persien, wo Gordon öfter war, aber das Exotische, Wilde, Ungezähmte findet eben nicht in den exotischen Ländern statt, denn Gordon hat in der Steppe Steppenwolf und Steppengeier nicht gesehen, sondern »im ganzen werden Sie die Bekanntschaft dieser liebenswürdigen Geschöpfe Gottes im Berliner Zoologischen Garten sichrer und kopierbarer machen als an Ort und Stelle« (30). Cécile und der Leser wissen das schon seit dem 1. Kapitel, denn dort deutet St. Arnaud aus dem Zugfenster und sagt: "Sieh, Cécile, das sind die Elefantenhäuser.« (8) Wie in Effi Briest Blut, Opfer und Heidentum immer wieder mitten in der preußischen Welt auftauchen, so wird in Cécile mindestens dem Hörensagen nach im königlichen Schloß selbst geschlachtet und eins der Opfer ist im übertragenen Sinne Cécile. »Die Reise nach dem Glück«, heißt es schon im 1. Kapitel, wird wohl »bloß ein Märchen« (10) sein. 
Der Hinweis auf die Perser und auf Wereschtschagins Bilder ist noch in einem anderen Zusammenhang aufschlußreich. Dieser Maler begleitete nämlich das russische Heer auf verschiedenen Feldzuigen in den Süden, in deren Verlauf die exotische vorderasiatische Welt zwischen Kaspischem Meer und China dem Hegemonismus Rußlands zum Opfer fiel. Im Roman auftauchende Namen wie General Skobelew, Plewna, Samarkand, Turkmenien gaben dem zeitgenössischen Leser, in dessen unmittelbarer Gegenwart diese Ereignisse stattfanden, deutliche Hinweise. Er kannte auch die Bilder Wereschtschagins, die 1882 und 1886 in Berlin ausgestellt waren und die auch Fontane besichtigt hat. In Cécile bildet die Nivellierung dieser exotisch-archaischen Welt durch Rußland eine deutliche Parallele zum gleichzeitigen Hegemonismus PreuBens, durch den die exotischen Duodezfürstentümer Deutschlands, aus deren Welt Cécile ein Relikt bildet, zum Untergang verurteilt wurden.

Auch Geschichte ist also in Fontanes Roman nicht Plauderei, ssmall talk<, sondern Deutungsmedium, und daher darf auch der sich in Cécile entfaltende Gegensatz zweier historischer Welten, des von mittelalterlicher Kaiserpracht zeugenden Harzes und des modernen Berlin der preußischen Könige und neuen deutschen Kaiser, nicht unbeachtet bleiben. Auch dieser Gegensatz war dem zeitgenössischen Leser viel gegenwärtiger und berührte ihn viel unmittelbarer. Er mußte an die damals viel diskutierte und zur Stellungnahme herausfordernde wissenschaftliche Fehde zwischen den Historikern Julius von Ficker und Heinrich von Sybel denken, die entgegengesetzt darüber urteilten, wie das Heilige Römische Reich Deutscher Nation vom modernen nationalen Standpunkt zu bewerten sei - eine Streitfrage, die im Gefolge der Entscheidung für das kleindeutsche Reich besonders aktuell war. Ficker sah im mittelalterlichen Kaisertum die Repräsentanz deutscher Interessen und die Habsburger Monarchie als Fortsetzung dieser Politik. Sybel verurteilte das Hegemonialstreben der mittelalterlichen Kaiser, weil diese imperiale Politik zur Schwächung Deutschlands geführt habe: "Das neue Kaisertum [Österreich] stand seiner Natur nach in ebenso schneidendem Widerspruch zu den Interessen der deutschen Nation wie das alte. ${ }^{14}$ Sybel begrüßte daher die Führungsrolle der preußischen Monarchie im kleindeutschen Reich. Sollte es sogar Absicht Fontanes sein, daß Cécile aus Schlesien stammt, dem Teil Österreichs, den Preußen im 18. Jahrhundert durch Friedrich II. erobert hatte?

Cécile ist geprägt durch die - paradoxerweise - amoralische, aber menschliche Welt des kleinfürstlichen Absolutismus, muß aber in einer Zeit leben, die sich - wieder ein Paradox - durch bürgerlich-moralische Unmenschlichkeit auszeichnet. Da Fontane alle ideologische Schwarzweißmalerei verhaßt war, darf man sich über solche Paradoxe bei ihm nicht wundern. Der Exponent der neuen Zeit ist Gordon, der Techniker, der in der ganzen Welt zu Hause ist und zum Erstaunen des Eselsjungen sogar im Himalaja war, der aber bei aller äußeren Unabhängigkeit gesellschaftlichen Konventionen unterworfen ist und 
dadurch seine Individualität aufgibt. Nicht umsonst wird er mehrmals mit seinem Namensvetter aus Schillers Wallensteins Tod in Verbindung gebracht, dem »Kommandant von Eger « (139), der schwankt, sich dem Urteil anderer fügt, nicht den Mut hat, die Katastrophe zu verhindern und sich über seinen aus Ängstlichkeit begangenen Verrat an Wallenstein mit dem Bewußtsein der erfüllten politischen Pflicht hinwegtröstet, anstatt seinem Herzen zu folgen. Es ist daher nur konsequent, daß er sein Schicksal von Butler-St. Arnaud bestimmen läßt.

Innstetten in Effi Briest spricht immer wieder von seiner Pflicht, die ihn zu bestimmten Handlungen zwingt, auch wenn sie ihn nicht überzeugen, und bekennt sich damit zu dem zentralen Begriff der Ethik des Preußen Kant und dem, was als typisch preußische Haltung empfunden wurde. Wie auffällig ist es, daß auch Gordon, als er sich von Cécile zu befreien beginnt, dieses zentrale preußische Wort im Munde führt: »Der Mensch lebt, um seine Pflicht zu tun und zu sterben.« (129) Der Schluß des Romans bildet eine ironische Umkehrung, aber es ist zu spät, als Cécile sich zu der »neuen Lehre bekennt, dem »Gefühl der Pflicht « (174), und Gordon einsieht, »wir glaubten und vertrauten nicht genug, und das sei der Quell all unsres Unglücks und Elends« (179).

In der neuen Welt, die von den Männern ihrer Umgebung beherrscht wird, kann Cécile nicht existieren, und die vergangene Zeit, der sie angehört, wird schon durch die Namen einiger Harzorte angedeutet, die sie besucht: Todtenrode und Altenbrak. Cécile geht zuletzt zugrunde, wie viele Gestalten in Fontanes Romanen, wenn sie in der Auseinandersetzung mit der Gesellschaft unterliegen. Es will so wenig zu dem populären Bild vom altersweisen Fontane passen, der angeblich das »heitere Darüberstehen « geradezu verkörpert, da $\beta$ von seinen 17 Romanen 7 mit Selbstmord enden und in 6 weiteren der unfreiwillige Tod der Hauptgestalten eine große Rolle spielt.

Das Frauenbild, dem Cécile entspricht, wird nicht mehr geschätzt. Sie hat in einer Welt gelebt, in der die Frau verwöhnt wurde und nach ihrem erotischen Reiz Ansprüche stellen konnte, und sie hat daher eine "ganz auf Huldigung und Pikanterie gestellte Natur « (41). Bildungswissen, das Cécile so sehr vermissen läßt, war dabei eher ein Mangel als eine Tugend, während jetzt Gestalten wie Eginhard Aus dem Grunde Preußen bevölkern, die aus nichts als Wissen bestehen und daher komische Züge haben. Damals bewarb sich der Mann um die Frau, indem er ihr huldigte, sie umcourte, und dies ist einer der Bereiche, wo der Wandel der Zeit sich in dem Roman am auffälligsten zeigt, denn Preußen hat einen neuen Typ von kollektiver Huldigung hervorgebracht. 
Dabei tritt der Mann nicht mehr als Individuum auf, sondern eingeordnet in eine militärische Formation. Man marschiert zur Huldigung auf und wieder ab. Von den »viele[n] Militärs (8) auf dem Potsdamer Bahnhof und den Gesangvereinen (Kap. 3) bis zu den vielen Offizieren im Theater in Berlin (Kap. 25), die Gordon als »reine Levée en masse « (166) bezeichnet, geistern Trupps von Männern durch das Buch. Ordnen und richten, formieren, kommandieren, präsentieren und salutieren sind die Vokabeln, mit denen etwa der Aufmarsch "der Turner in Drillichanzügen « beschrieben wird, von denen wieder Gordon sagt: "Das ist Deutschland. Jung-Deutschland.« (83) Daß genau in dieser Szene beim Rezitieren der Leberreime St. Arnauds Beitrag von Rosa Hexel kommentiert wird mit der Bemerkung: »Ach, das ewig siegreiche Militär, siegreich auf jedem Gebiete. In neuester Zeit auch (leider) auf dem der Malerei. Doch das sind trübe Betrachtungen « (98) unterstreicht, wie der preußische Militarismus zum Zeichen der Zeit geworden ist: »Preußischer Drill und Gedächtnisballast.« (76)

Aber es zeigt sich beim Schmerlenessen auch auf peinliche Weise, wie es die Männer verlernt haben, der Frau zu huldigen, denn keiner von ihnen kommt auf den Gedanken, aus dem geforderten Vers ein Kompliment für Cécile zu machen, so daß Rosa es schließlich selbst anmerkt und die schöne Frau als »der Perlen schönste Perle« (99) feiern muß. Ähnlich abgeneigt zur Huldigung sind auch die beiden Berliner Touristen, wenn sie bei der Frage „Wen lassen wir leben, den Stöpsel oder die Stricknadel?« (35) die Wahl zwischen Cécile und Rosa diskutieren und dann zu der Entscheidung kommen, auf keine von beiden zu trinken, sondern statt dessen auf Berlin, die preußische Hauptstadt, die auf diese Weise ganz deutlich zum Gegenpol, zur Konkurrenz Céciles und der Frauen überhaupt wird, weil sie sie ihres angestammten Rechts auf Huldigung beraubt. Daß Gordon sich zuletzt bei und nach dem Theaterabend regelrecht daneben benimmt, ist die letzte Steigerung dieses den ganzen Roman durchziehenden Motivs der verweigerten Huldigung.

$\mathrm{Da}$ die neue Zeit auch einen neuen Frauentyp hervorgebracht hat, wird dem Leser durch Rosa Hexel und Baronin Snatterlöw zu Bewußtsein gebracht. Beide haben sprechende Namen, und beide gehören, obwohl sie durchaus verschiedene Menschentypen sind, darin zusammen, daß ihnen das Erotische fehlt. Die Baronin ist eine Art Mannweib, und mit Rosa könnte Gordon, so sagt er, »um den Äquator fahren« (154), ohne in Versuchung zu geraten. Sie erfährt das Heikle nicht in der Menschenwelt, sondern als Tiermalerin, denn das ist als Beruf »an der Grenze des Unerlaubten. Es gibt da so viele intrikate Dinge« (27). Aber schon ihr Name deutet auf ihre mangelnde erotische Anziehungskraft hin, die mit Cécile kontrastiert. Rosa ist die abgeblaßte Form der Liebesfarbe rot, und da sie Hexel - die Verkleinerungsform von Hexe - heißt und am Engel-Ufer wohnt, worauf Fontane durch Céciles 
Frage »wie kann man am Engelufer wohnen« (154) ausdrücklich hinweist, verfehlt sie das Weibliche nach oben oder unten, ist Engel oder Hexe, nicht Frau. Der Hexentanzplatz - wieder ein Motiv des Romans -, der so viele Gefahren für Cécile birgt, kann ihr deshalb auch nicht gefährlich werden. Sie steht als Künstlerin außerhalb der Konventionen und der menschlichen Tragik (»Und ich habe durchaus keine Schultern für das Tragische«, 87) und ähnelt damit - um wieder auf Fontanes bekanntesten Roman als Parallele hinzuweisen - Marietta Trippelli aus Effi Briest.

Wenn sich der schönen Cécile in der männlichen Welt die menschliche Welt versagt, weil die männliche Welt in sich selber kreist - St. Arnaud verbringt seine Tage im Club, und Gordon besucht nicht umsonst in Berlin einen Industriellen -, wenn dem Frauentyp, den sie repräsentiert, in einer zunehmend von Militär und militärischen Verhaltensweisen beherrschten Gesellschaft das Lebenselixier entzogen wird, bleibt ihr nur die Natur, und tatsächlich überschüttet diese sie, worauf ihr Mann wiederholt hinweist, mit Huldigungen: Fliederbouquet, der Hund Boncoeur, »eine Wolke von Rosenblättern« (59), und "gelbe Schmetterlinge, massenhaft« (73) werden von ihr geradezu magisch angezogen. Aber wie lange kann Cécile sich noch auf die Blumenteppiche verlassen, die ihr zu Füßen liegen? Auch hier ist die neue Zeit, in der der Profit herrscht und Menschliches nach Prinzipien übers Knie gebrochen wird, schon eingezogen: Die »endlos scheinende[n] Blumenbeete « in Quedlinburg dienen schon anderen Zwecken. Es gibt dort »zwei große Gartenfirmen«, die mit »Blumensamenpaketen ein Vermögen erworben « (40) haben, wie ähnlich die Nathusiusse, von denen auf der Fahrt nach Quedlinburg die Rede ist. Wo einst die reichsunmittelbaren Fürstäbtissinnen residierten, haben jetzt Preußen und der Kapitalismus Einzug gehalten.

Überhaupt darf das Quedlinburg-Kapitel, das ein Musterbeispiel bildet für Fontanes Kunst der versteckten Symbolik, für seine Fähigkeit, das Zentrale im scheinbar Marginalen zu verbergen, bei der Diskussion dieser Aspekte des Romans nicht übergangen werden, weil es der Ort ist, wo die beiden Welten, die alte aristokratische Zeit Céciles und die neue bürgerliche Zeit Preußens, aufeinanderstoßen, wo ihr Nebeneinander so greifbar ist wie die Statuen der durch ihre eigene Leistung berühmt gewordenen Bürgerlichen, die jetzt im fürstlichen Tiergarten stehen: »der berühmte Geograph« (37) Ritter und der Dichter Klopstock.

Von dem Geburtshaus dieser bürgerlich-emanzipatorischen Gestalt des 18. Jahrhunderts weiß Cécile nichts und will sie nichts wissen. »Es ist so grün« (41), ist das einzige, was sie darüber zu sagen hat. Aber mit dieser scheinbar 
unsinnigen Bemerkung wird ein weiterer Bereich von Symbolen in die Deutung der Geschichte einbezogen: Farben, die bisher in der Fontane-Forschung fast vollständig übergangen worden sind. Mit dem unerotischen, Ruhe verbreitenden Grün wird die bürgerliche Bildungswelt Klopstocks zur Gegenwelt Céciles und der toten Fürstäbtissinnen (»daß es nichts Toteres gibt als solche Galerie beturbanter alter Prinzessinnen«, 47). Fast unmittelbar vor der Bemerkung über das Klopstock-Haus wird an den Blumen das Rot als Brennende Liebe identifiziert, und im fürstlichen Schloß herrscht dieses Rot der Liebe und der aristokratischen Macht - man denke nur an den Purpur - in den »roten Damasttapeten « (43) und am Thron der Äbtissinnen, »ebenfalls rot, aber von rotem Samt und mit Hermelin verbrämt « (44). Das Eigenartige aber an diesem Schloß, das von Cécile und ihrem Kreis besichtigt wird, ist seine Leere. Es konnte, »durch alle Räume hin, als eine wahre Musterniete gelten«. Der Schloßführer muß deshalb »den herkömmlichen, an vorhandene Sehenswürdigkeiten anknüpfenden Kastellansvortrag in einen umgekehrt sich mit dem Verschwundenen beschäftigenden Geschichtsvortrag « (43) umwandeln. Die Bedeutung dieser immer wieder erwähnten Leere ist klar: Die Welt der Fürstäbtissinnen und der Fürstengeliebten wie Cécile ist untergegangen. Geblieben ist nur eine süffisante Art, darüber zu reden, die für Cécile eine ständige Verlegenheit bedeutet.

Aber mit dem leeren Schloß ist wieder ein sich durch den Roman ziehendes Motiv getroffen, denn öfter wird Cécile bei ihrem Harzaufenthalt mit verödeten Häusern konfrontiert. Erst begegnet sie (Kap. 5) in der »mit wildem Wein und Efeu dicht überwachsene[n] Villa [...] einer absolut unbewohnten Stätte «, wo der Anstrich von „verwunschene[m] Schloß« und »Idyll« (23) über die grausame Wirklichkeit von Blut und verlorenem Glück nicht hinwegtäuschen kann - beides auch Elemente von Céciles eigenem Schicksal. An ihre aristokratische, vergangene Welt erinnern Pfau, Rittersporn und Brennende Liebe im wuchernden Garten der Villa - Zeichen, denen der Leser an anderen Stellen des Buches wiederbegegnet. Später (Kap. 13) kommt Cécile mit Gordon auf dem Weg nach Altenbrak an »drei ziemlich gleich aussehende[n] Häuschen « vorbei, die wieder als »verwunschen « bezeichnet werden und wieder "ganz von wildem Wein«, dem Zeichen von Unordnung, Freiheit, Rausch, überwachsen sind. Innen gibt es »keine Gardine, kein[enj Vorhang, überhaupt nichts, was auf Bewohnerschaft hätte deuten können« (84 f.), aber Cécile erfährt mit tiefem Unbehagen, daß hier ein alter Herzog "Bacchus, und dann Eros« (86) zu opfern pflegte. Wieder ist die Beziehung zu ihrer eigenen Vergangenheit deutlich.

Diese Vergangenheit und der Wandel der Zeit, der sie so anstößig macht, sind in Quedlinburg immer gegenwärtig. Der Parvenükönig Jérôme Bonaparte hat das Mobiliar des Schlosses verschleudert; den König von Preußen hat die Bevölkerung mit ihren »Bourgeoismanieren« aus Quedlinburg vertrieben; der 
großartige Kristallspiegel der Fürstinnen, in dem Céciles Schönheit sich hätte spiegeln können, ist verschwunden wie fast alle Zeugnisse des aristokratischen Lebens, und daß sie »mechanisch und ohne $\mathrm{zu}$ wissen, was sie tat, an die Wandstelle klopfte, wo der Kristallspiegel seinen Platz gehabt hatt« (46), stellt ihr unbewußtes Vermissungserlebnis eindringlich dar und macht dem Leser ihre Selbstverliebheit bewußt, die sich auch darin ausdrückt, daß »ihre Augen [...] scharf nach innen [standen], wie wenn sie sich suchten und lieber sich selbst als die Außenwelt sähen« (52). Wenn sich das Gespräch bei der Schloßbesichtigung auf die Fürstengeliebten konzentriert, über die Gordon in zynischem Ton spricht, wird Cécile auf immer neue Weise gequält.

Und so enthüllt sich auch die Geschichte vom Regensteiner als eine Spiegelung von Céciles Schicksal, denn im Gegensatz zur gängigen Auffassung wird für den gefangenen Ritter Partei genommen, »und die liberale Geschichtsschreibung greift in nichts so fehl, als darin, daß sie den Bürger immer als Lamm und den Edelmann immer als Wolf schildert«. So wie der Ritter von den Quedlinburger Bürgern gefangen und ausgestellt wurde, denn »die Bourgeoisie, die nie tief aus dem Becher der Humanität trank, war gerade damals von einer besonderen Abstinenz«; so wie er von der Bevölkerung "mit ihren Sonnenschirmen« (38) gepiekt wurde, ist auch Cécile ständiger Neugier und Zudringlichkeit in der bürgerlichen Welt ausgesetzt, wird auch sie ständig gepickt. Sogar der Sonnenschirm ist nicht zufällig. Mit ihm hat ihrerseits am Anfang des Romans Cécile die Siegessäule zu picken versucht, und das Quedlinburg-Kapitel beginnt mit dem Satz: »Die Sonne brannte heiß auf den Perron nieder.« (39) Diese »wahre Tropensonne« (40) deutet - wie auch in anderen Romanen Fontanes - darauf hin, daß etwas in gnadenloses Licht gebracht wird oder die Helle des Tages nicht scheut. Cécile wird Wahrheiten ausgesetzt, von denen sie sich erst in der "prächtig kühlen Kirche« (49) erholen kann - auch dies ein Aspekt ihres Lebens, denn sie sucht schon seit längerem in einer Kirche den Trost, den ihr der preußische Protestantismus nicht geben kann. Auch hier ist der Versuch ihrer Borussifizierung gescheitert.

Noch der Schluß des Quedlinburg-Kapitels ist voller Anspielung: »je mehr Don Juan, je mehr Torquemada« (48), also je mehr Weiberheld, desto inquisitorisch strengere Moralvorstellungen. Gordon wird auf diese Weise durch historische Figuren beleuchtet, und wieder öffnet sich hier ein ganzer Bereich von Spiegelungen in Fontanes Roman.

Mehrmals werden die Hauptgestalten durch historische oder künstlerische Figuren reflektiert. Die Personen in Mozarts Don Giovanni dienen wiederholt - wie hier - als Spiegel des Geschehens. Cécile wird zum Beispiel zu Maria Stuart, die ebenfalls ihrer mit sexueller Schuld beladenen Vergangenheit nicht entgehen kann, und Gordon zu Wagners Tannhäuser in Beziehung gesetzt, der 
zwischen dem Venusberg und der heiligen Elisabeth von Thüringen schwankt.

Hier wie auch in so vielen anderen Motivbereichen kann der Leser seine Kombinationsgabe spielen lassen, um weitere Bezüge zu entdecken. Er wird immer wieder die zu Anfang erwähnte Erfahrung machen, daß jeder Roman Fontanes ein außerordentlich dichtes Gewebe von Verweisen darstellt. Die Natürlichkeit seiner Szenen, die Zwanglosigkeit des Dialogs, der typisch Fontanesche Plauderton sind nicht Harmlosigkeit und Oberflächlichkeit, sondern enthalten ständig Signale für den Leser, bilden einen höchst kritischen Zeitkommentar und folgen strengen Kunstgesetzen.

Für den vorliegenden Roman bestätigt das Verfolgen solcher Motive schon Conrad Wandrey, der 1919 in der ersten Gesamtdarstellung von Fontanes literarischem Werk sagte: "Cécile bleibt trotz der Ansiedlung im Harz ein >Berliner Roman $<. \ll{ }^{15}$

Nichts wäre Fontane weniger gemäß, als das Gegeneinander der beiden Welten und moralischen Konzeptionen zu einem Schwarzweißbild zu machen. Fontane hatte zum Ideologen kein Talent und für Ideologen keine Achtung. Er beobachtete die Zeichen der Zeit; ihm ging es um Ambivalenzen des bürgerlichen Tugendideals der Frau, das sich im 18. Jahrhundert entwickelt und - so schien es Fontane - eine Wandlung des Rollenverhaltens von Mann und Frau mit sich gebracht hatte.

Die Frau verliert an erotischem Reiz in dem Maß, in dem sie moralisch beurteilt wird. Erscheint sie als emanzipiertes Wesen wie Rosa Hexel, dann bezahlt sie die Unabhängigkeit vom Mann offenbar mit dem Verlust ihrer erotischen Anziehungskraft. Veraltet wird man Fontanes Beobachtungen wohl nicht nennen können. Wie die Frau dann darauf verzichtet, dem Mann als erotischer Lebensbezug zu dienen, macht umgekehrt der Mann das Soldatische, eine rein männliche Tätigkeit, zu seiner ausschließlichen Daseinsform. Sie durchdringt die ganze Gesellschaft und wird als preußischer Militarismus $\mathrm{zu}$ einem soziologischen Phänomen. Uniform werden damit auch die moralischen Bewertungskategorien, die den einzelnen Menschen zur Schablone stempeln und unterwerfen. Daß St. Arnaud und Gordon den Soldatenberuf aufgeben, zeigt symbolisch, daß sie diesen Verlust der Individualität ablehnen und der Frau ihren angestammten erotischen Platz im Leben des Mannes lassen wollen. St. Arnaud hat Cécile gegen den Druck der Konvention erkämpft, und Gordon umcourt sie. Aber da beide zugleich von den neuen Bewertungen durchdrungen sind, landen sie in einem Vakuum, in dem sie desorientiert sind und desorientierend auf die Frau wirken, weil sie in Verhalten und Handeln 
inkonsequent und daher unberechenbar sind. Cécile konnte kaum ahnen, daß St. Arnaud sie in der Ehe so behandeln und Gordon seine Einstellung ihr gegenüber so abrupt ändern würde. Gordon verurteilt im Moralischen zunächst das Schwarzweißbild durchaus, dem er dann doch zum Opfer fällt, und er formuliert dabei eine psychologische Einsicht, die Fontane selbst sicher aus der Seele gesprochen ist: »Die Welt ist eine Welt der Gegensätze, draußen und drinnen, und wohin das Auge fällt, überall Licht und Schatten. Die dankbarsten Menschen überschlagen sich plötzlich in Undank, und die Frommen, mit dem seligen Hiob an der Spitze, murren wider Gott und seine Gebote. Was hat nicht alles Platz in einem Menschenherzen? Alles verträgt sich, man rückt mit Gut und Bös ein bißchen zusammen, und wer heute sittlich ist und morgen frivol, kann heute gerade so ehrlich sein wie morgen.« (159) Die bürgerliche Gesellschaft holt Gordon wider seinen Willen ein.

Cécile versucht dem bürgerlichen Alltag ganz zu entgehen, und dieses Element ihres Lebens ist, wie so vieles, schon im 1. Kapitel bei der Abfahrt des Zuges aus Berlin gegenwärtig, wenn Fontane die »merkwürdige[n] Dinge « andeutet, die man vom Zug aus in »offenstehenden Schlafstubenfenstern«, »Sommergärten und Vergnügungslokale[n]«. (8) sehen kann - Gegenbilder zu den leeren aristokratischen Häusern im Harz; und auch dort wird Cécile vor dem Alltag abgeschirmt. Zweimal schlägt Gordon ihr vor, einen Weg zu nehmen, wo man »Hütten, Kinder und aufgehängte Wäsche« (22) nicht sieht, beziehungsweise die »Mischluft von Küchenabguß und Pferdeställen [...] Menschen und Butterpapiere[n], Krüppel und Ziehharmonika« (67) meiden kann. Wenn Gordon sich dem alltäglichen Leben nahe fühlt und auf dem Wochenmarkt sogar »alles primitiv, aber entzuickend in seiner Heiterkeit und Farbe « (117) findet, ist Cécile nicht in Berlin, sondern auf ihrem Gut, dem Geschenk ihrer fürstlichen Gönner.

Wie in mehreren anderen Fontane-Romanen drückt sich das Recht des Individuums auf seine Stimme auch am Schluß von Cécile im erzählerischen Perspektivismus aus: In Gordons letzten Zeilen an Cécile, in dem sachlichen Zeitungsbericht über das Duell, in St. Arnauds Brief aus Mentone an seine Frau, in Céciles Testament, in dem sie - nach dem Vorbild von Schillers Maria Stuart - in rührenden Gesten ihren irdischen Besitz verteilt und das selbst wieder einen Teil von Hofprediger Dörffels Brief an St. Arnaud bildet, werden dem Leser die verschiedensten Deutungen angeboten. Er hat mit seinem Abwägen das letzte Wort über die Ereignisse. 
Anmerkungen

${ }^{1}$ AHR 4.563.

${ }^{2} \mathrm{DuD} 2.345$.

${ }^{3} \mathrm{HB} 3.390$.

${ }^{4} \mathrm{DuD} 2.348$

${ }^{5}$ AHR 4.570 .

${ }^{6}$ Nach Auskunft des Theodor Fontane Archivs (Potsdam), wo das Original des Briefes sich befindet, wäre eher $>25<\mathrm{zu}$ lesen, was sich aber schlecht mit Gordons Militärdienst und beruflichen Erfahrungen vereinbaren ließe.

${ }^{7} 25$. April, wohl 1885 (DuD 2.349).

82. Juni 1887 (HB 3.539)

${ }^{9}$ An seine Frau, 25. Juni 1883 (HB 3.264)

${ }^{10}$ An dies., 21. März 1880 (HB 3.68).

${ }^{11}$ An Georg Friedlaender, 30. Mai 1893 (Friedlaender Briefe, S. 221).

${ }^{12}$ An Emil Dominik, 14. Juli 1887 (HB 3.551).

${ }^{13}$ Theodor Fontane, Effie Briest - Ein Leben nach christlichen Bildern. Tübingen 1978, S. $10 \mathrm{ff}$.

${ }^{14}$ Die deutsche Nation und das Kaiserreich. Eine historisch-politische Abhandlung. Düsseldorf 1862, S. 87.

${ }^{15}$ Wandrey, S. 199. 